\title{
QoS Evaluation of Real-Time Applications over a Multi-domain DiffServ Experimental Test-Bed
}

\author{
G. Carrozzo, V. Chionsini, S. Giordano, and S. Niccolini \\ Department of Information Engineering University of Pisa \\ Via Diotisalvi 256126 Pisa Italy Tel. +39 050 568511, Fax +39050 568522 \\ \{g.carrozzo,v.chionsini,s.giordano,s.niccolini\}@iet.unipi.it
}

\begin{abstract}
This paper presents a QoS evaluation in a DiffServ experimental testbed scenario. We implemented our field trial using prototypal routers running under Linux OS and we arranged them in order to make possible the interconnection with a remote island of a Multi-domain DiffServ network. The performance evaluation of Real-time applications presented in the paper will make clear how it is possible to provide "mission critical" applications with tool-quality level of service when appropriate algorithm and resource sharing are chosen and when these features are associated with a fair degree of aggregation. As a consequence the paper describes the results by means of a Mean Opinion Score (MOS) evaluation campaign to show how Real-Time applications (such as voice and video conferencing) may suffer for the lack of QoS.
\end{abstract}

Keywords: Experimental test-bed, Multi-Domain, DiffServ, Real-Time traffic.

\section{Introduction}

During the past years different proposals for Next Generation Internet architecture have been suggested. Integrated Services [1] and Differentiated Services [2] were the most promising ones. Unfortunately both of them showed their weakness when dealing with end-to-end QoS guarantees (in particular, IntServ lacks of scalability, and DiffServ lacks of "hard" guarantees). This research work intends to show how, by means of simple DiffServ mechanism applying to prototypal routers (edge and core DiffServ routers), it is possible to obtain satisfying results in terms of QoS parameters and in terms of user perceived quality. The IntServ access network is supposed to be unchanged compared with the framework of IntServ over DiffServ architecture [3]. The rest of the paper is organized as follows: in Section 1 we present our design and implementation of a real Multi-Domain DiffServ experimental test bed carried out in the framework of NEBULA project [4]. We show how DiffServ mechanism and our DiffServ aggregation strategies [5] well behave when dealing with Real-time application (mostly voice and video). In Section 3 the discussion is about the treatment of audio and video within the Real-time class. In Section 4 we analyze and comment the results highlighting the goodness of our aggregation strategies assumption. Finally we present our conclusion and future works. 


\section{Test-Bed Description}

This section presents our Multi-Domain test-bed, built-up in order to study the obtainable performance of a DiffServ Core Network when appropriate QoS mechanisms are used. We emulated three simple access domains interconnected by a DiffServ cloud by means of an ATM link in order to arrange the trial to be remotized. Our implementation is related to multiple site interconnection, in order to insert our trial in a more complex network (as in the scope of NEBULA project), where the study of QoS performance is more critical. The access domains are emulated by means of source and destination PCs connected to separate private networks. In Fig. 1 we detail our field trial; it is possible to distinguish the access domains where the sources and the destinations are located.

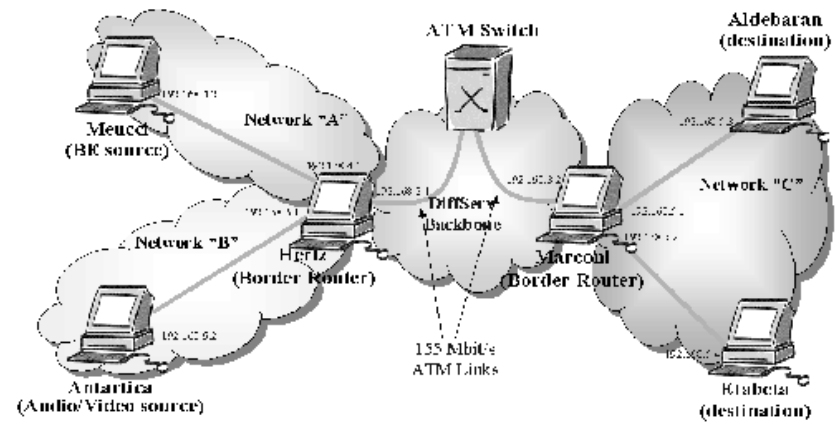

Fig. 1. Field Trial

We developed our field trial under Linux OS running on IA32 platforms (PC). The interconnecting routers are equipped with two 10/100 Ethernet cards and one MMF ATM card; each source/destination PC has only one 10/100 Ethernet card and it is point-to-point connected to the Border Router. The DiffServ backbone is emulated by means of an ATM connection (i.e. two $155 \mathrm{Mbit} / \mathrm{s}$ links towards a Newbidge CS1000 ATM switch). The Border Routers (Hertz and Marconi) provide the necessary transformation from packets to cells and viceversa by means of the AAL5 protocol. We implemented, on the BRs, the DiffServ traffic control functionalities (i.e marking, shaping, metering, dropping), by means of the "TC" package available under Linux. The scheduler used by the BRs is a CBQ (Class Based Queuing) algorithm.

\section{Real-Time Traffic \& Non Real-Time Traffic}

The traffic used in this field trial may be classified in two main classes: a) Real time traffic: we include both audio and video sources because both of them have strict bounds on QoS target, even if different statistical features; b) Non Real-Time traffic: we include both MGEN (UDP source) traffic and FTP traffic (TCP source) because nor of them requires strict bounds on delay/jitter. The first test, whose results are pre- 
sented in Section 4 provided a simple distinction between the two mentioned classes. The adopted combination between scheduling algorithm and TC functionalities had the aim to protect the Real-Time class against an "aggressive" and "persistent" BE class, formed by UDP sources. The second test, according to evaluations derived from a previous work [5], provided a refined classification, in order to avoid performance degradation experimented when voice and video flows are merged together.

\section{QoS Evaluation}

In all the tests presented in this section, Real-Time traffic was sent across the network. The first test conducted on the DiffServ backbone was about the flow isolation obtainable using the marking/scheduling algorithm on two classes (the first group of test is related to the transport of audio or video on the EF class while the rest of the traffic is forwarded on the BE class). We have collected the QoS relevant parameters directly between ingress and output interface of the ingress border router (named Hertz in Fig. 1), this because of synchronization problems arising from an end-to-end collection of delay.

Table 1. DiffServ EF configuration (video on EF)

\begin{tabular}{|c|l|c|l|}
\hline Flow & \multicolumn{1}{|c|}{ Flow Description } & ToS & \multicolumn{1}{|c|}{ CBQ Class Parameters } \\
\hline 1 & Video: $384 \mathrm{kbit} / \mathrm{s} ;$ Avg Packet size $=800 \mathrm{Bytes}$ & EF & Buffer $=3.2 \mathrm{kB} ;$ Rate $=390 \mathrm{kbit} / \mathrm{s}$ \\
\hline 2 & MGEN: rate $=1 \mathrm{Mbit} / \mathrm{s} ;$ packet size $=1 \mathrm{kB}$ & $\mathrm{BE}$ & Buffer $=60 \mathrm{kB} ;$ Rate $=500 \mathrm{kbit} / \mathrm{s}$ \\
\hline
\end{tabular}

In the first test the scope was comparing QoS parameters of Real-Time Traffic, in terms of rate, delay and MOS in two cases: a) No DiffServ: all the traffic was sent on the same queue and served in a FIFO way; b) DiffServ: configuration described in Table 1. In Fig. 2 it is possible to notice that the portion of bandwidth used by the video flow increases when the protecting DiffServ scheduling scheme is activated. Fig. 3 shows that the performance degradation is more evident when speaking about the delay measurement. When the DiffServ is disabled all the flows share the same class and so the delay experimented by the video packets is the same experimented by the BE packets. On the other hand, when the video flow has its own queue (DiffServ enabled) we obtain the required service differentiation. In order to deeply analyze the performance of the video related to this test we have conducted a MOS (Mean Opinion Score) campaign; a MOS campaign is a collection of user sensation about quality perception by means of numerical score ( $1=$ lowest quality, $5=$ highest quality). This campaign has allowed to evaluate the perceived user quality at application level. We report in Fig. 6 a) the MOS evaluation obtained from the campaign when two video coding rates were considered: $128 \mathrm{kbit} / \mathrm{s}, 384 \mathrm{kbit} / \mathrm{s}$. From the results it is possible to notice the application level performance improvement when DiffServ architecture is enabled. 

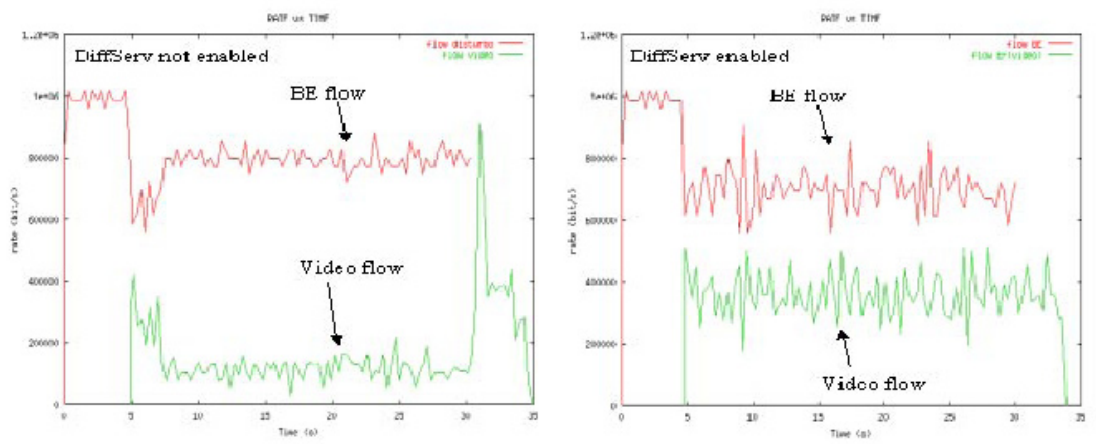

Fig. 2. Traffic rates: DiffServ disabled and enabled
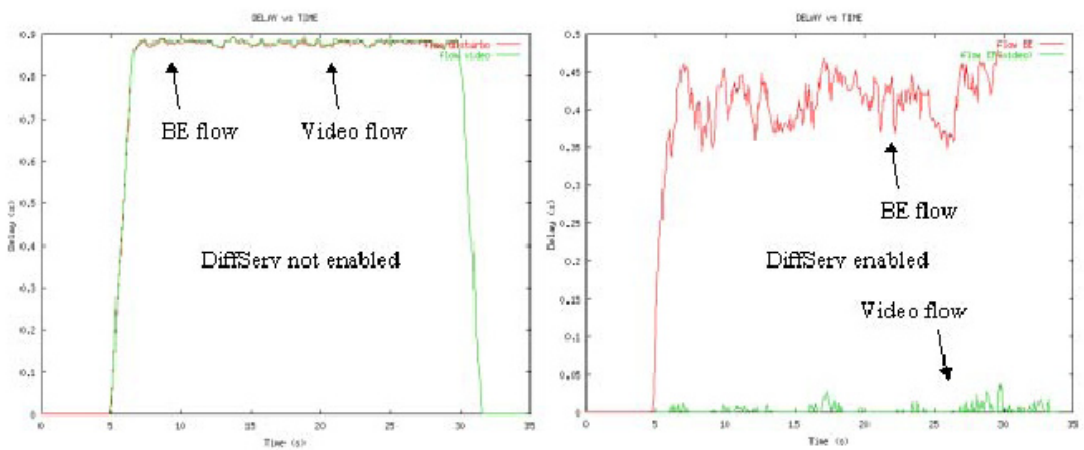

Fig. 3. Enabling the DiffServ mechanism on the video flow (delay)

A second test group is mainly focused on the evaluation of the impact of different aggregation strategies on the QoS parameters at network level and on the user perceived quality at application level. We will compare a scenario where only one class (EF) is used to carry Real-time traffic to a second scenario where we use separate classes in order to carry Real time flows (voice on EF and video on AF). The BE class is used in both cases to carry non-RT traffic. In Table 2 we show the scheduling parameters used in this test group.

Table 2. EF audio, AF video configuration

\begin{tabular}{|c|l|c|l|}
\hline Flow & \multicolumn{1}{|c|}{ Flow Description } & ToS & \multicolumn{1}{|c|}{ CBQ Class Parameters } \\
\hline 1 & Audio: $64 \mathrm{kbit} / \mathrm{s} ;$ Packet size $=172$ Bytes & EF & Buffer $=1 \mathrm{kB} ;$ Rate $=64 \mathrm{kbit} / \mathrm{s}$ \\
\hline 2 & Video: $128 \mathrm{kbit} / \mathrm{s} ;$ Avg Packet Size $=800$ Bytes & AF & Buffer $=15 \mathrm{kB} ;$ Rate $=128 \mathrm{kbit} / \mathrm{s}$ \\
\hline 3 & MGEN: rate $=1$ Mbit $/ \mathrm{s} ;$ packet size $=1 \mathrm{kB}$ & BE & Buffer $=60 \mathrm{kB} ;$ Rate $=500 \mathrm{kbit} / \mathrm{s}$ \\
\hline
\end{tabular}

The EF configuration adopted when audio and video are carried together may be obtained simply adding the CBQ parameters (i.e. Buffer $=16 \mathrm{kB}$ and Rate $=192 \mathrm{kbit} / \mathrm{s}$ ). We adopted this configuration in order to perform a fair comparison in terms of allocated resources. Fig. 4 shows the delay experimented by traffic flows when audio is carried on $\mathrm{EF}$ and video on $\mathrm{AF}$, all collected on the DiffServ ingress BR. In this case 
the delay experimented by the audio flow is $35 \mu \mathrm{s}$ (we had to zoom the statistic in order to make its visualization clearer) while the video delay is much more relevant (mean value: $25 \mathrm{~ms}$ ). Video flow experimented a mean delay lower than BE flow one but higher than audio one, because of its different service class (AF) and its intrinsic burstiness. Anyway, the absolute values should not be considered, because they are relative to the crossing of a single device.

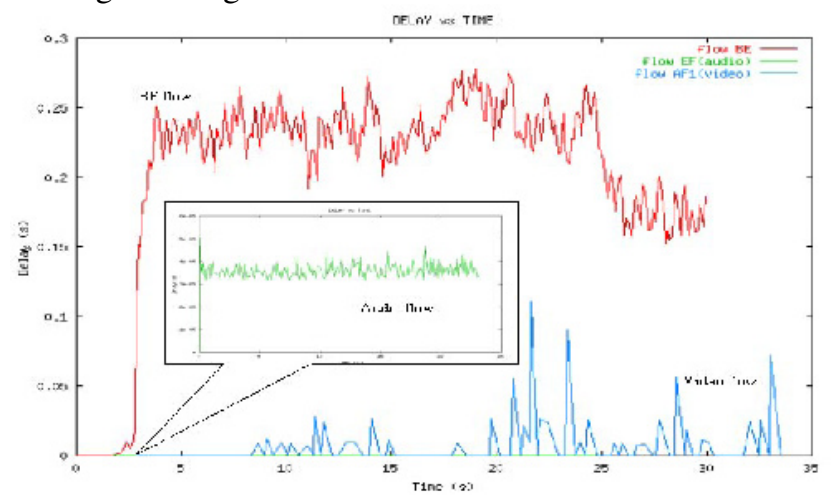

Fig. 4. Collection of delay parameter on BR (Audio on EF, Video on AF)

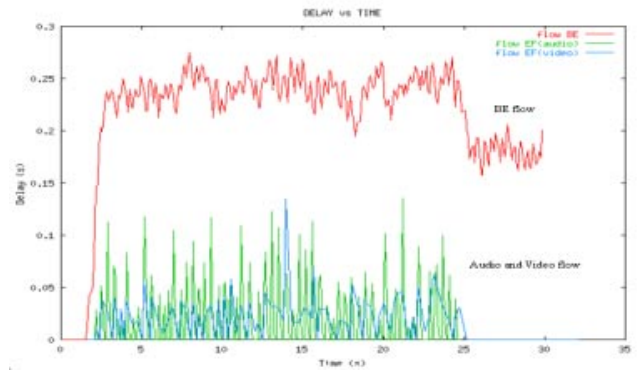

Fig. 5. Collection of delay parameter on BR (Audio and Video on EF)
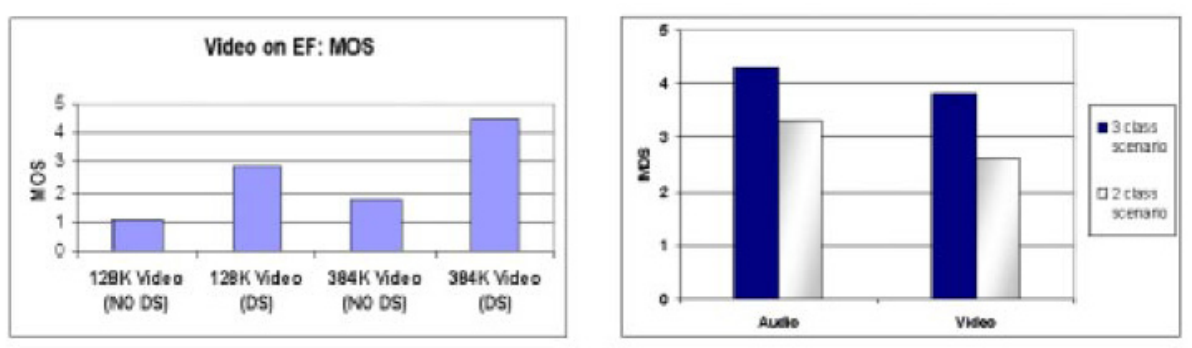

Fig. 6. MOS evaluation: a)Video on EF; b)Audio/Video with different aggregation strategies

When audio and video are merged together (EF class) there is a degradation of the audio performance: being carried together with the video, it suffers the same order of 
delay (see Fig. 5). At last we present in Fig. 6(b) the MOS evaluation collected in order to make a comparison between our proposed two-classes aggregation scheme and one-class aggregation scheme. Fig. 4 compared to Fig. 5 highlights the better performance obtained with the two-classes aggregation scheme. As it concerns the application level QoS, the MOS shown in Fig. 6(b) takes benefit from the forwarding of audio and video on two separate PHBs. As it can be noticed, the gap is approx. one point of MOS scale between the two scenarios: it is a great degradation of quality when speaking about user perceived quality at application level.

\section{Conclusion and Ongoing Works}

The main goal of the paper was the QoS evaluation of Real-time applications over a Multi-Domain DiffServ experimental test-bed by means of network level QoS parameters and application level parameters. In this framework the we have presented a two-classes aggregation scheme for DiffServ architecture in order to improve the obtainable performance. The collected results in the experimental test-bed scenario demonstrate they were satisfactory both at application level (evaluated by means of a MOS campaign) and at network level (evaluated by means of rate/delay statistics). This work is a first extract of our ongoing work on developing a real Multi-domain DiffServ island interconnection. A deeper analysis of end-to-end delays experimented in such a scenario is going to be conducted by means of a host synchronization tool (GPS system).

Acknowledgments. The authors wish to thank Andrea Giorgi for his help in this work. This work was partially supported by the project "NEBULA" of the Italian MURST.

\section{References}

1. R. Braden et al., "Integrated Services in the Internet architecture: An overview", RFC 1663, June 1994.

2. S. Blake et al., "An architecture for Differentiate Services”, RFC 2475, December 1998.

3. Y. Bernet et al. "A Framework for Integrated Services Operation over Diffserv Networks", RFC 2998, November 2000.

4. NEBULA Project, financed by the Italian MURST (http://cofin98.cineca.it/murst-dae/).

5. R.G. Garroppo, S. Giordano, S. Niccolini, F. Russo, "A Simulation Analysis of Aggregation Strategies in a WF2Q+ Schedulers Network" in Proceedings of The 2nd IP Telephony Workshop, New York, April 2001. 